\title{
Embedded method of optoelectronics education in electrical engineering curriculums
}

\section{Alexander Poularikas}

Alexander D. Poularikas, "Embedded method of optoelectronics education in electrical engineering curriculums," Proc. SPIE 2525, 1995 International Conference on Education in Optics, (13 October 1995); doi: $10.1117 / 12.224031$

SPIE Event: SPIE's 1995 International Symposium on Optical Science, Engineering, and Instrumentation, 1995, San Diego, CA, United States 


\title{
The Embedded Method of Optoelectronics in Electrical Engineering Curriculums
}

\author{
by \\ Alexander D. Poularikas \\ Electrical and Computer Engineering \\ University of Alabama in Huntsville, Huntsville AL, 35899
}

\begin{abstract}
Modern electrical engineering students need to learn about any new emerging field that directly impacts and is important to their profession. The development of the low-loss fibers, the miniature laser/detector systems, the photonic switches, the nonlinear optical devices, the optical signal processing, etc., have created the need to incorporate this special new knowledge into electrical engineering curriculum. However, the curriculum is restricted to a specific number of credit hours and, in most cases, it is impossible to add new courses unless other courses, which may be important, are deleted. To alleviate this problem, some 15 years ago Professor Seely and the author proposed the embedded method as a solution to the problem. This method is flexible, can be adopted at any level of instruction, can be incorporated in any field within the electrical engineering discipline, is easily implemented, and can also be adopted by any other field outside engineering which needs such a modification.
\end{abstract}

\section{Rationale}

In the same way that the invention of the transistor initiated the modern electronics era, the nearly simultaneous development of low-glass optical fiber and the recent semiconductor laser/detector systems initiated the photonics area. Within the past few years, long-haul telecommunications have become dominated by light wave systems. Research laboratories are engineering systems based on III-V materials to manipulate photons in some of the same sophisticated ways that silicon systems manipulate electrons. Such systems and devices are referred to as photonics systems and devices. Parallel development of other materials, such as nonlinear optical organic materials, show great promise for providing a basis for sophisticated and inexpensive devices. Compact, robust passive optical systems have been demonstrated that would have been regarded as impossible only a few years ago. Practical optical amplifiers based on erbium-doped glass fibers are now commercial products. It seems inevitable that the key technologies for transmitting and processing information will soon be based on the manipulation of photons, rather than electrons. Many, if not most, of these systems will be integrated hybrids of photonic and electronic devices, that is, optoelectronic devices. 
It is essential that institutions of higher education must be prepared to provide the knowledge required to incorporate emerging photonics technologies into society. The rapid development of laser sources, optical fibers, and semiconductor optoelectronic devices has lead to an abundance of applications that directly impact our every day experience. A growing photonics industrial base assures employability of graduates from the field of optoelectronics. These industries include lasers, laser sources into optical memories, precision optics, laser diodes, and fiber and guided wave optics.

In addition to existing employment opportunities, trends indicate that photonics applications will enhance or displace many existing electronic and mechanical technologies. Prime examples of this trend are:

- The information carrying capacity of optical fibers, perhaps using solitons, will displace copper cable systems in delivering computer and video information to the home and office. The eventual displacement of copper wire will require design and manufacture of millions of photonic devices.

- As mechanical and electronic devices shrink in scale, current manufacturing and measurement methods will become inadequate. Optical and photonic based techniques will be their replacements.

- $\quad$ Fiber and photonic devices will foster a new generation of industrial and commercial sensors. As an example, common engineering materials can be equipped with extremely small stress sensors using optical fibers.

\section{The Problem}

Electrical Engineering is concerned broadly with the generation, transmission, processing, and control of electromagnetic and electronic signals. There has been a continuous technological evolution from electromechanical devices to vacuum devices to solid state devices, and from applications beginning at low frequencies, then extending to radio and microwave frequencies, and now moving heavily into the optical domain. This evolution has influenced electrical engineering curriculums in both the key underlying device technologies and the applications that are emphasized. It is apparent that we must stress the continuation of this trend into the optical domain with the resultant need for inclusion of optical phenomena, devices, and applications in electrical engineering core courses as well as in selected devices.

The growing importance of optics in applications indicates a strong need to incorporate optoelectronics into electrical engineering curriculums. The methods that have been tried to remendy the problem are:

1. Incorporate courses on optoelectronics into existing electrical engineering core courses.

2. Offer optoelectronic elective courses for undergraduate electrical engineering students. 
3. Offer optoelectronic options (well-defined curriculums for students wishing to specialize in optoelectronics) within existing electrical engineering curriculums.

4. Develop independent degree programs in optoelectronics.

Immaterial what is the new field that must be introduced into electrical engineering curriculums, and this applies equally well to any other discipline, the basic problem that has faced engineering educators during the last half of the 20th century is how to adapt their particular curriculums to the technical developments that have occurred during this ensuing period. It has been our experience and general observation that the usual approach is to rearrange course content, by changing course requirements, and by substituting a new sequence of courses, as it was outlined above for the specific case of optoelectronics, often at the expense of basic courses covering the principles on which general understanding depends. Often these methods have caused communications gaps, since they assume a level of student sophisticationat a given time, which may not have been attained in previous studies. All to frequently the student must tell the professor that the assumptions that he had previously studied certain material basic to the new program is not valid. This lack of sequence might result from educational gaps that are inherent within departmental offerings. Therefore, the educator must find solutions that satisfy the educational needs of the students, incorporate the new knowledge, and accomplish all these with a restricted number of credit hours.

\section{Proposed Solution}

The we propose is the embedded method of instruction. The basic philosophy of this method is the splitting of an area into sub-areas and introduce the sub-areas into different standard courses that exist in the curriculum. The material can be incorporated in different forms such as problems, examples, footnotes, text, figures, etc. To investigate the effectiveness of our proposed curriculum changes, we are considering the important area of optoelectronics in the electrical engineering curriculum.

\section{Previous Efforts by the Author}

Granting that the optoelectronics is a very important area that our undergraduate students' intellect must enrich, Professor Seely and I have tried in the past 15 years to implement our ideas on embedded method and communicate them to our colleagues across the country. Primarily we have confined ourselves into writing texts and incorporating the appropriate material such that the idea of embedded method is implemented.

Based on the average electrical engineering curriculum, we have identified three basic areas that are taught in every electrical engineering department. These are: Electromagnetics, Signals, and Communications. To implement our method we wrote two books, each one containing the appropriate topics from the field of optics. These are: 


\section{Topics from Optics}

Geometric and Physical Optics

Fiber Optics $\underline{\text { E.E Courses }}$

ELECTROMAGNETIC THEORY:

¿Electromagnetics Classical and Modern

Theory and Applications.

S. Seely and A. D. Poularikas, 720 p.

Marcel Dekker, New York, NY, 1979]

SIGNALS AND SYSTEMS:

[Signals and Systems. A. D. Poularikas and S. Seely. 2nd Edition, 1010 p. PWSKENT, Boston, MA, 1992]

COMMUNICATIONS

[To be written]

Optical Communication

Sources and Detectors

Shot Noise

Optical Modulation

In our Electromagnetic Theory book we have introduced the following topics from the field of optics:

\section{CRYSTAL OPTICS}

Fermat's principle

Propagation of optical beams

Image formation in Gaussian

optics

Diffraction holography

Fabry-Perot resonator optics

\section{GEOMETRIC OPTICS}

Imaging in refractive media

Fiber optics

Diffraction optics

Gaussian light beam

Optical resonators

\begin{abstract}
Aberration coefficients
Rays guided by lenses

Fraunhofer region and

Coherence of fields

Stability diagram of

resonators
\end{abstract}

In the Signals and Systems book we have introduced the following topics from the field of optics:

Fourier transforms of optical systems

Optical system functions

Optical transfer function

Frequency response of incoherent system

Optical correlation

Vander Lugt filter
Modulation of light

Spread function

Frequency response of coherent system

Optical filtering

Phase and amplitude filters 
Our philosophy on education and the proposed embedded method have been received with enthusiasm by the academic community. This conclusion is based on the many reviews and adoptions of our two books.

In addition to the three main areas in which the microelectronics can be embedded, there are also the Semiconductor Devices and Electronic Circuits.

Many photonic components are made of semiconductor materials. Inclusion of these devices in a beginning semiconductor course is natural and essential in a modern electrical engineering program. The course should cover solid state sources and detectors.

Transmitters and receivers for optical communications links are, basically, electronic circuits incorporating photonic devices. Simple circuits of this type can easily be inserted into the conventional electronics circuits courses. The suggested topics include driver circuits for laser diodes and light-emitting diodes, photodetector receiver circuits, and repeaters and equalizers. With the tremendous impact of fiber optics on communications, any modern course on the topic should include a description of the properties of the fibers as a transmission medium-bandwidth, dispersion, coherence versus incoherent carrier sources, and shot noise. Other topics that should be included are: optical modulation, modulators and optical receivers.

\section{Implementation}

The plan consists of five the following five parts:

1. A national survey will be conducted to find out what the average form of instruction the new areas of optoelectronics.

2. Development of the appropriate text material of optoelectronics to be embedded in the different courses, as mentioned above.

3. Develop optical experiments for demonstration purposes.

4. Develop optical experiments for students who are interested to satisfy their senior project in the area of optoelectronics.

5. Assemble videos, software and other pedagogical material that will enhance student's understanding of the physical phenomena and concepts that he/she is exposed.

\section{Conclusions}

The embedded method approach will alleviate the problem of almost regularly having to introduce new courses into the EE curriculum and at the same time will satisfy a great pedagogical need, the need to show that learning the fundamentals is paramount in education and that one can use the information in many seemingly diverse fields. 
The embedded method is: a) Flexible, since you can add and or subtract material as new items become more important, b) Universal, since it can be applied to any discipline old or new, c) Highly pedagogical, since it brings in the fundamentals of any area of study, d) Interdisciplinary, since the material of one area appears in many areas of study. Figures 1 and 2 depict the optical areas to be embedded and the EE curriculum.

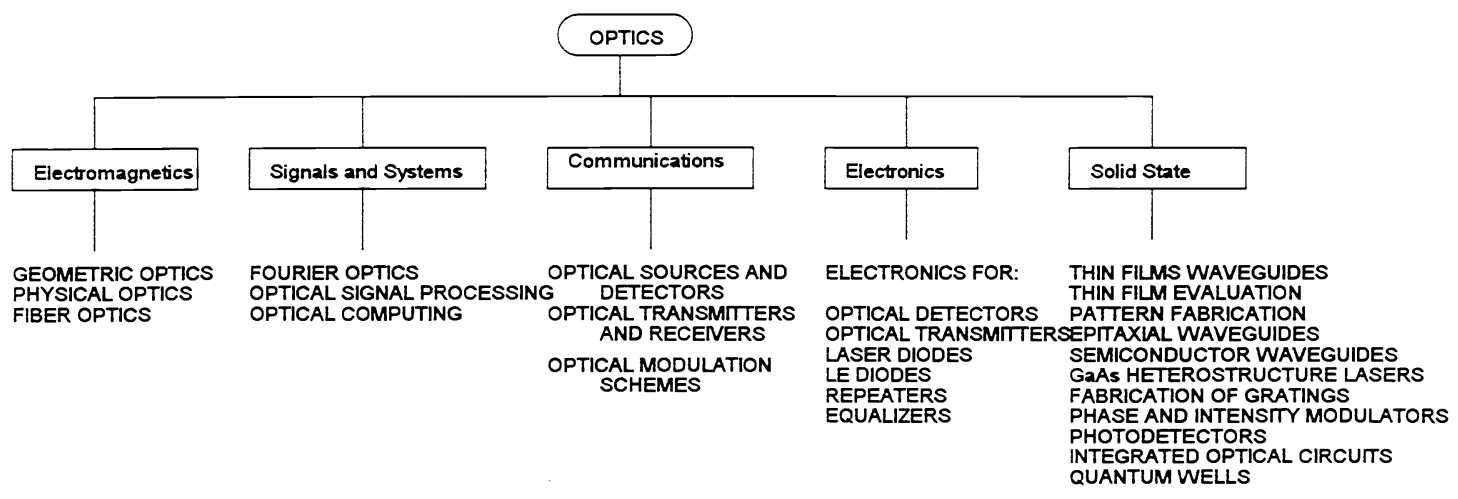

Figure 1.

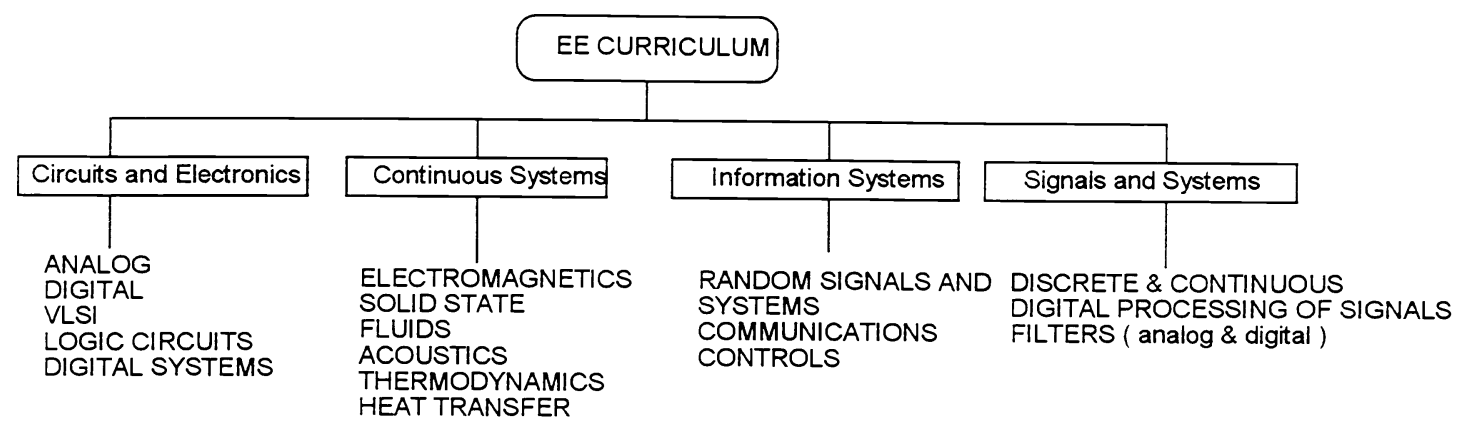

Figure 2 . 